\title{
Avaliação da reprodutibilidade da classificação de Dejour para instabilidade femoropatelar*
}

\section{Evaluation of the Reproducibility of the Dejour Classification for Femoropatellar Instability}

\author{
Rodrigo de Souza Mendes Santiago Mousinho ${ }^{1}$ José Neias Araújo Ribeiro ${ }^{2}$ \\ Francisco Kartney Sarmento Pedrosa ${ }^{1}$ Diego Ariel de Lima ${ }^{1,2,3,4}$ Romeu Krause Gonçalves ${ }^{1}$ \\ José Alberto Dias Leite 2,3
}

${ }^{1}$ Instituto de Traumatologia e Ortopedia Romeu Krause (Itork), Recife, PE, Brasil

2 Universidade Federal do Ceará, Departamento de Ortopedia e Traumatologia, Fortaleza, CE, Brasil

${ }^{3}$ Centro Universitário Christus (Unichristus), Fortaleza, CE, Brasil

${ }^{4}$ Universidade Federal Rural do Semiárido, Mossoró, RN, Brasil
Address for correspondence Diego Ariel de Lima, Universidade Federal Rural do Semiárido, Mossoró, RN, Brasil (e-mail: arieldelima.diego@gmail.com).

Rev Bras Ortop 2019;54:171-177.

\section{Resumo \\ Palavras-chave \\ - articulação femoropatelar \\ - instabilidade articular \\ - reprodutibilidade}

Objetivo Avaliar, pela reprodutibilidade interobservador e intraobservador, a classificação proposta por David Dejour para descrever a displasia troclear do joelho.

Métodos Foram estudados dez pacientes com diagnóstico de displasia troclear. Três médicos membros da Sociedade Brasileira de Cirurgia do Joelho foram convidados para avaliar as imagens. Análises intra- e interobservador foram feitas com intervalo de uma semana. A reprodutibilidade foi avaliada em quatro cenários: uso de radiografia; uso de radiografia e tomografia; uso de radiografia, consultando-se a classificação no momento; e uso de radiografia e tomografia, consultando-se a classificação no momento.

Resultados A avaliação intraobservador apresentou resultados discordantes. Na análise interobservador, o grau de concordância foi baixo para as análises que usavam apenas a radiografia e excelente para aquelas que associavam radiografia e tomografia. Conclusão A classificação de Dejour apresentou uma baixa reprodutibilidade intra e interobservador quando usada somente a radiografia em perfil. Demonstrou-se que o uso apenas da radiografia para classificar pode gerar falta de uniformidade até mesmo entre observadores experientes. Contudo, quando radiografia e tomografia foram associadas, a reprodutibilidade melhorou.

\footnotetext{
Abstract

Objective To evaluate the classification proposed by David Dejour to describe trochlear dysplasia of the knee through inter- and intraobserver reproducibility measurements.
}

\footnotetext{
Trabalho desenvolvido no Departamento de Ortopedia e Traumatologia, Centro Universitário Christus (Unichristus), Fortaleza, CE, Brasil. Originalmente publicado por Elsevier Ltda.
}

received

September 14, 2017

accepted

November 21, 2017
DOI https://doi.org/

10.1016/j.rbo.2017.11.003. ISSN 0102-3616.
Copyright $\odot 2019$ by Sociedade Brasileira License terms de Ortopedia e Traumatologia. Published by Thieme Revnter Publicações Ltda, Rio de Janeiro, Brazil 


\section{Keywords}

- femoropatellar joint

- joint instability

- reproducibility
Methods Ten patients with trochlear dysplasia were studied. Three physicians, members of the Sociedade Brasileira de Cirurgia do Joelho (Brazilian Society of Knee Surgery), were invited to evaluate the images. Intra- and interobserver analyses were performed at one-week intervals. Reproducibility was evaluated in four scenarios: using only radiography; using radiography and tomography; using radiography and consulting the classification; and using radiography and tomography, consulting the classification.

Results The intraobserver evaluation presented discordant results. In the interobserver analysis, the degree of agreement was low for the analyses that used only radiography and excellent for those in which both radiography and tomography were used.

Conclusion The Dejour classification presented a low intra- and interobserver reproducibility when only the profile radiography was used. It was demonstrated that the use of the radiography alone for classification may generate lack of uniformity even among experienced observers. However, when radiography and tomography were combined, reproducibility improved.

\section{Introdução}

A patela, conhecida como o maior osso sesamoide do corpo humano, é fundamental na biomecânica da articulação do joelho. Tem as funções de aumentar o poder mecânico do aparelho extensor e proteger a articulação do joelho. ${ }^{1}$

A síndrome patelofemoral é um termo usado para descrever uma condição na qual o trilhamento patelar entre os côndilos femorais ocorre de forma inadequada. Esse deslizamento impróprio provoca dor anterior no joelho, pode levar a alterações degenerativas ou luxação/instabilidade da articulação femoropatelar. ${ }^{2}$

A instabilidade femoropatelar é uma patologia frequente e ligada a fatores predisponentes na maioria dos pacientes. Dentre tais, a displasia da tróclea femoral e a altura da patela são consideradas os fatores mais importantes. ${ }^{3}$

A instabilidade patelar é mais comum entre mulheres jovens, entre os 10 e 17 anos. A taxa de luxação após o primeiro episódio varia de $15 \%$ a $44 \%$ após tratamento conservador, essa taxa é maior em quem teve mais de um episódio. ${ }^{4}$

A displasia troclear é caracterizada por morfologia troclear anormal e sulco "raso". Não está claro se displasia troclear é causa ou consequência da instabilidade. Ou seja, alterações congênitas poderiam levar à displasia da tróclea, que seria menos profunda e que favorecesse a instabilidade; ou se alteraçõesmusculares determinariam um trilhamento anormal da patela, reduziriam a pressão femoropatelar, o que geraria um estímulo inadequado ao desenvolvimento da anatomia da tróclea e a tornaria mais plana e displásica; ou se causada por uma combinação de fatores. ${ }^{3,5}$

Exames de imagem apresentam muitos sinais, permitem a identificac,ão de grande e de pequenas anormalidades anatômicas, auxiliam no estabelecimento do planejamento do tratamento. ${ }^{6-9}$ Imagens radiográficas em incidência lateral são fundamentais para avaliar e classificar a displasia troclear e para quantificar a patela alta. Incidências axiais permitem a medic,ão dos ângulos da linha intercondilar e de congruência. ${ }^{10}$ Imagens tomográficas podem permitir a definic,ão da distância TT-LITAGT, o valor da inclinação e as características rotacionais e avaliar a displasia troclear. ${ }^{11} \mathrm{~A}$ ressonância é valiosa na luxação aguda e pode mostrar uma ruptura do ligamento patelofemoral medial, além de lesões osteocondrais e contusões ósseas. ${ }^{12}$

A displasia troclear foi bem avaliada e classificada através de exames de imagem por Dejour. ${ }^{13}$ Auxiliado por radiografia e tomografia computadorizada, Dejour ${ }^{14}$ classificou a displasia em tipos A, B, C e $D^{15}$ (- Fig. 1).

Como vimos acima, a classificação de Dejour é importante para o tratamento do paciente com a referida displasia. Todavia, uma característica que deve estar presente em qualquer classificação e sua reprodutibilidade. ${ }^{16}$ Para tanto, tal classificação deve ser simples, de fácil memorização e auxiliar na escolha do tratamento, orientar o prognóstico e facilitar a comunicação entre os profissionais da saúde. ${ }^{16}$

Assim, o principal objetivo do presente estudo é avaliar, através da medida da reprodutibilidade interobservador e intraobservador, a classificação proposta por David Dejour ${ }^{14}$ usada para descrever a displasia troclear do joelho.

\section{Material e Métodos}

O estudo foi aprovado pelo Comitê de Ética com número CAAE 67648217.3.0000.5049. Todos os participantes estavam de acordo e assinaram o termo de consentimento livre e esclarecido entregue antes do início. Não foi oferecido incentivo financeiro para que participassem e os voluntários poderiam se recusar a participar do estudo, ou retirar seu consentimento a qualquer momento, sem precisar justificar.

Foram selecionados no ambulatório 10 sujeitos aleatoriamente, sem restrição de sexo ou idade, com diagnóstico de instabilidade femoropatelar. Todos os pacientes fizeram exames radiográficos para acompanhamento de suas patologias. 

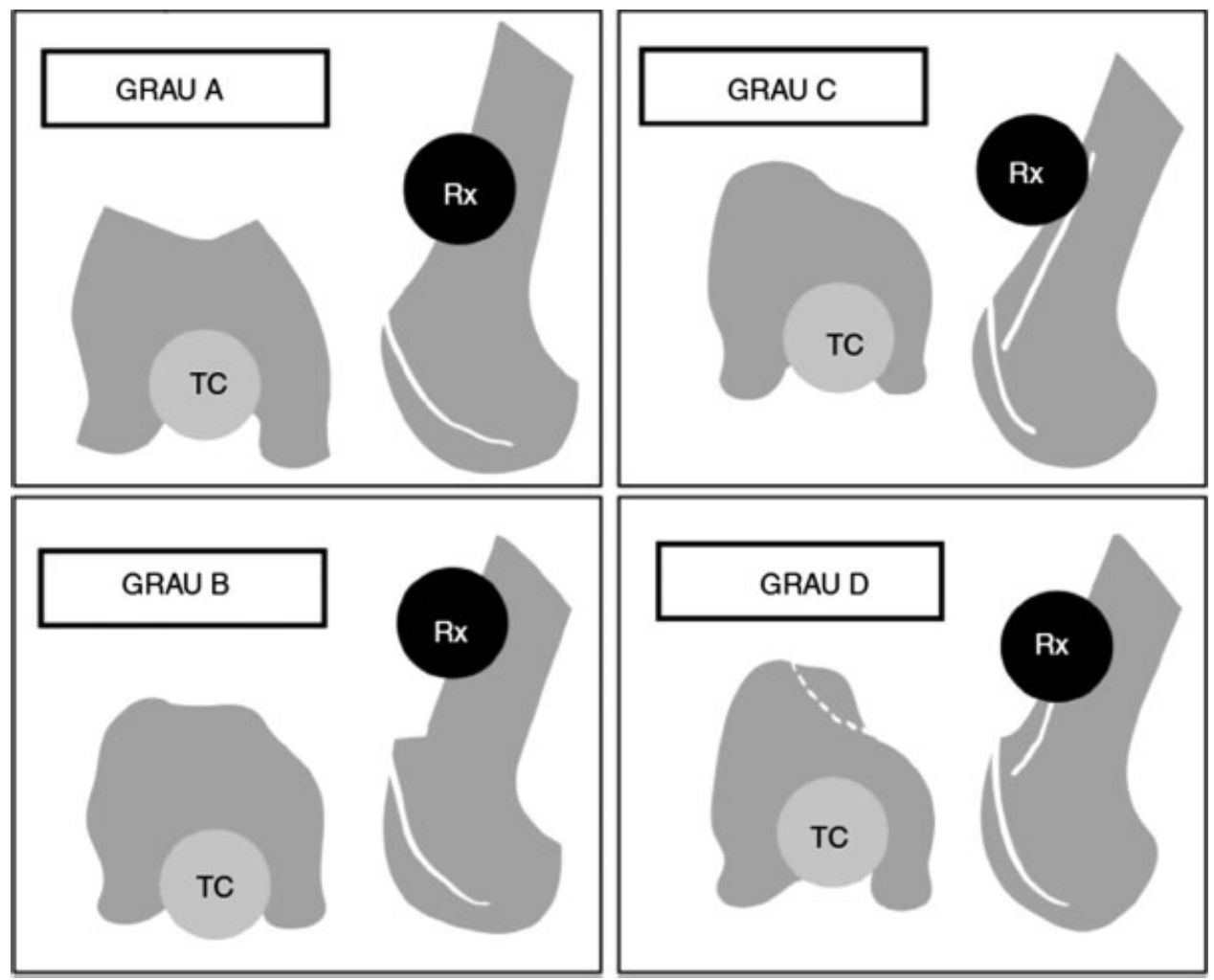

Fig. 1 Classificacão de Displasia Troclear de Dejour ${ }^{14}$ (radiografia e tomografia). Abreviações: Rx, raio X; TC, tomografia computadorizada.

Nenhum exame de imagem adicional foi solicitado neste estudo. Foram usados apenas os que os participantes já tinham.

Solicitamos aos sujeitos da pesquisa uma cópia de seus exames de imagens mais recentes: uma radiografia em incidência em perfil e uma tomografia computadorizada em cortes transversais do joelho acometido pela patologia e que tenham sido gravados em mídia digital, como CD-ROM.

Os critérios de exclusão foram: indivíduos que relatassem cirurgia prévia do joelho em questão e que não tivessem imagens radiológicas anteriores a esse procedimento. Após aplicação dos critérios, nenhum participante foi excluído.

Para analisar as imagens, foram convidados três observadores membros da Sociedade Brasileira de Cirurgia de Joelho. As imagens digitalizadas foram entregues em um CD-ROM (copiadas depois da autorização dos pacientes) aos observadores. A fim de minimizar o viés, devido à dificuldade de interpretação ou possível esquecimento, a classificação e suas variantes estão descritas na - Fig. 1. As análises radiográficas foram feitas de forma cega e precedida de uma revisão da classificação momentos antes (-Fig. 1). Os três examinadores, separadamente e sem contato com outros examinadores, avaliaram as imagens dos 10 sujeitos alocados. Primeiramente classificaram (Dejour A, B, C ou D) apenas com a radiografia em perfil (Análise 1) e depois com a tomografia e radiografia (Análise 2).

Após uma semana, as mesmas imagens, randomizadas, foram examinadas pelos mesmos avaliadores. Primeiramente classificaram (Dejour A, B, C ou D) apenas com a radiografia em perfil (Análise 3 ) e depois com a tomografia e radiografia (Análise 4 ).
Logo em seguida a essa segunda avaliação, os mesmos observadores classificaram novamente com a radiografia em perfil e depois com a tomografia, mas dessa vez podiam consultar a classificação no momento da avaliação (Análises 5 e 6$)$.

As variações inter e intraobservador dos dados tabulados foram analisadas pelo software SPSS (Statistical Package for the Social Sciences), v23, SPSS, Inc. Foram consideradas significativas as comparações com valor de p até 0,05 , com intervalo de confianc,a de 95\%. Para análise de concordância entre os avaliadores foi usado o coeficiente de concordância W de Kendall (-Tabela 1). Foram categorizados em escala ordinal com a classificação de Dejour, na qual $\mathrm{A}=1 / \mathrm{B}=2 / \mathrm{C}=3 / 4=\mathrm{D}$.

\section{Resultados}

A variação interobservador foi calculada a partir de seis situações: Análise 1: avaliação da classificação com apenas radiografia; Análise 2: avaliação com radiografia e

Tabela 1 Coeficiente de concordância W de Kendall avalia o grau de concordância entre os avaliadores, de ruim a excelente concordância

\begin{tabular}{|l|l|}
\hline W de Kendall & Interpretação \\
\hline$<0,4$ & Ruim \\
\hline $0,400-0,599$ & Regular \\
\hline $0,600-0,800$ & Bom \\
\hline$>0,8$ & Excelente \\
\hline
\end{tabular}


174 Avaliação da reprodutibilidade da classificação de Dejour Mousinho et al.

Tabela 2 Variação interobservador nas seis situações propostas

\begin{tabular}{|l|l|l|l|l|}
\hline Situação & W de Kendall & Qui-quadrado & GL & Significância assintótica (p) \\
\hline Análise 1 (Rx) & 0,553 & 14,931 & 9 & 0,093 \\
\hline Análise 2 (Rx + TC) & 0,891 & 24,058 & 9 & 0,004 \\
\hline Análise 3 (Rx) & 0,515 & 13,903 & 9 & 0,126 \\
\hline Análise 4 (Rx + TC) & 0,861 & 23,238 & 9 & 0,006 \\
\hline Análise 5 (Rx + consulta) & 0,606 & 16,354 & 9 & 0,060 \\
\hline Análise 6 (Rx + TC + consulta) & 0,883 & 23,840 & 9 & 0,005 \\
\hline
\end{tabular}

Abreviações: GL, graus de liberdade; Rx, raio X; TC, tomografia computadorizada.

tomografia; Análise 3: reavaliação após uma semana, com apenas radiografia; Análise 4: reavaliação após uma semana, com radiografia e tomografia; Análise 5: avaliação com radiografia e consulta à classificação no momento; Análise 6: avaliação com radiografia e tomografia e consulta à classificação no momento (-Tabela 2 e - Fig. 2).

A variação intraobservador foi expressa em valores de concordância W de Kendall entre os três avaliadores para cada tipo de avaliação (-Tabela 3 e - Fig. $\mathbf{3}$ ).

\section{Discussão}

Classificar as patologias é prática comum, principalmente na ortopedia e traumatologia. Um bom sistema de classificação tem por finalidade ser simples, reprodutível e capaz de agrupar diferentes estágios de uma lesão em subgrupos homogêneos e permitir comparações, algoritmos de tratamento e prognóistico. ${ }^{16} \mathrm{O}$ que geralmente acontece com as classificações é que, ao longo do tempo, aparece um caso que não se enquadra nos tipos descritos ou classificados. Assim, algumas classificações ao longo do tempo foram substituídas por outras mais completas. ${ }^{17}$
A estabilidade da articulação femoropatelar é de grande importância para o funcionamento adequado do mecanismo extensor do joelho e da sua articulação como um todo. ${ }^{18}$ No entanto, ela tem um baixo grau de congruência, conforme estabelecido pelo equilíbrio da arquitetura óssea e das restrições de tecidos moles. Alterações anatômicas não são raras e, como resultado do desequilíbrio mecânico, pode ocorrer instabilidade. A apresentação clínica da instabilidade, no entanto, apresenta um espectro de manifestações. Assim, é importante diferenciar os pacientes que têm sintomas, mas que não apresentam anormalidades anatômicas, daqueles que têm subluxação e/ou luxação. ${ }^{19}$

Segundo DeJour et al, ${ }^{6}$ destacam-se quatro principais fatores anatômicos que levam à instabilidade ${ }^{6,20}$ :

1. Displasia da Face Patelar ou Displasia Troclear: o formato da face patelar é anormal e a contenção óssea do desvio da patela é perdida;6,20

2. Distância entre a tuberosidade da tíbia e "garganta" da tróclea femoral (TT-LITAGT) excessiva: situação associada ao mau alinhamento do mecanismo extensor, com a produção de um vetor em valgo que age sobre a patela;6,20

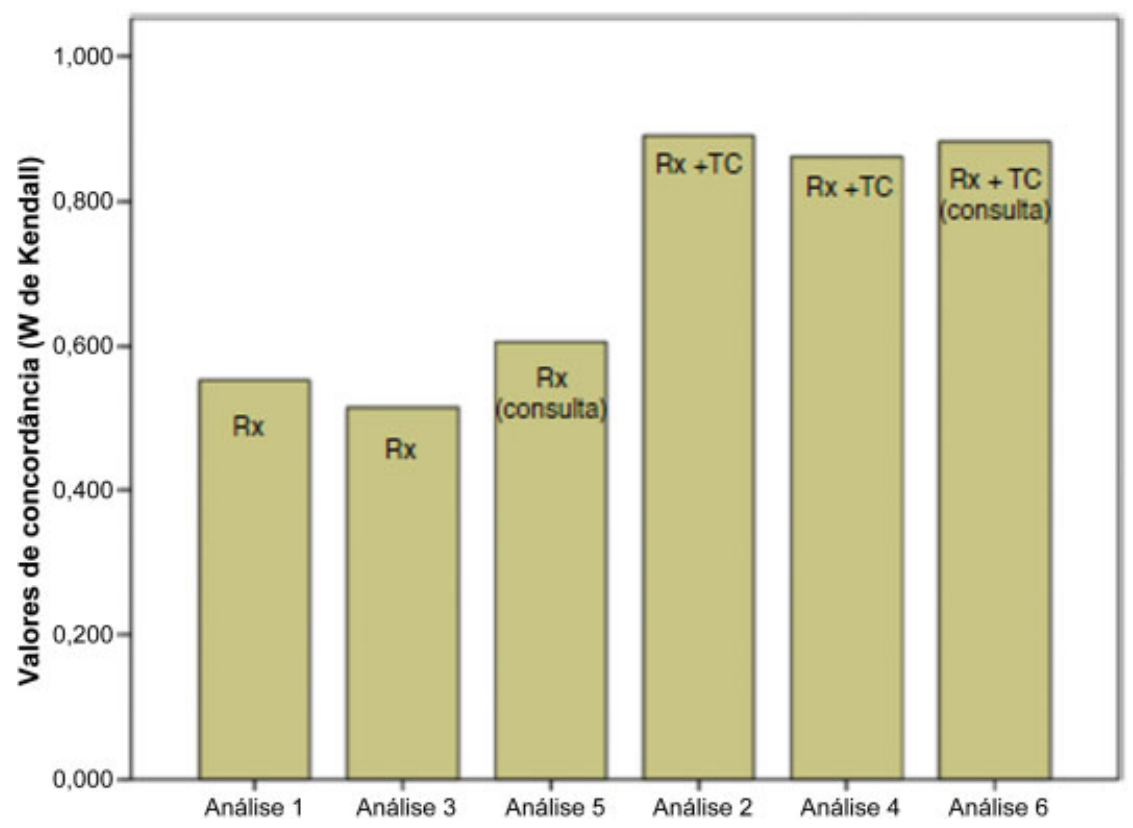

Fig. 2 Variação interobservador nas seis situações propostas. 
Tabela 3 Variação intraobservador

\begin{tabular}{|l|l|l|l|l|}
\hline & W de Kendall & Qui-quadrado & GL & Significância assintótica (p) \\
\hline Avaliador 1 & 0,532 & 28,716 & 9 & 0,001 \\
\hline Avaliador 2 & 0,873 & 47,143 & 9 & 0,000 \\
\hline Avaliador 3 & 0,397 & 21,422 & 9 & 0,011 \\
\hline
\end{tabular}

Abreviações: GL, graus de liberdade.

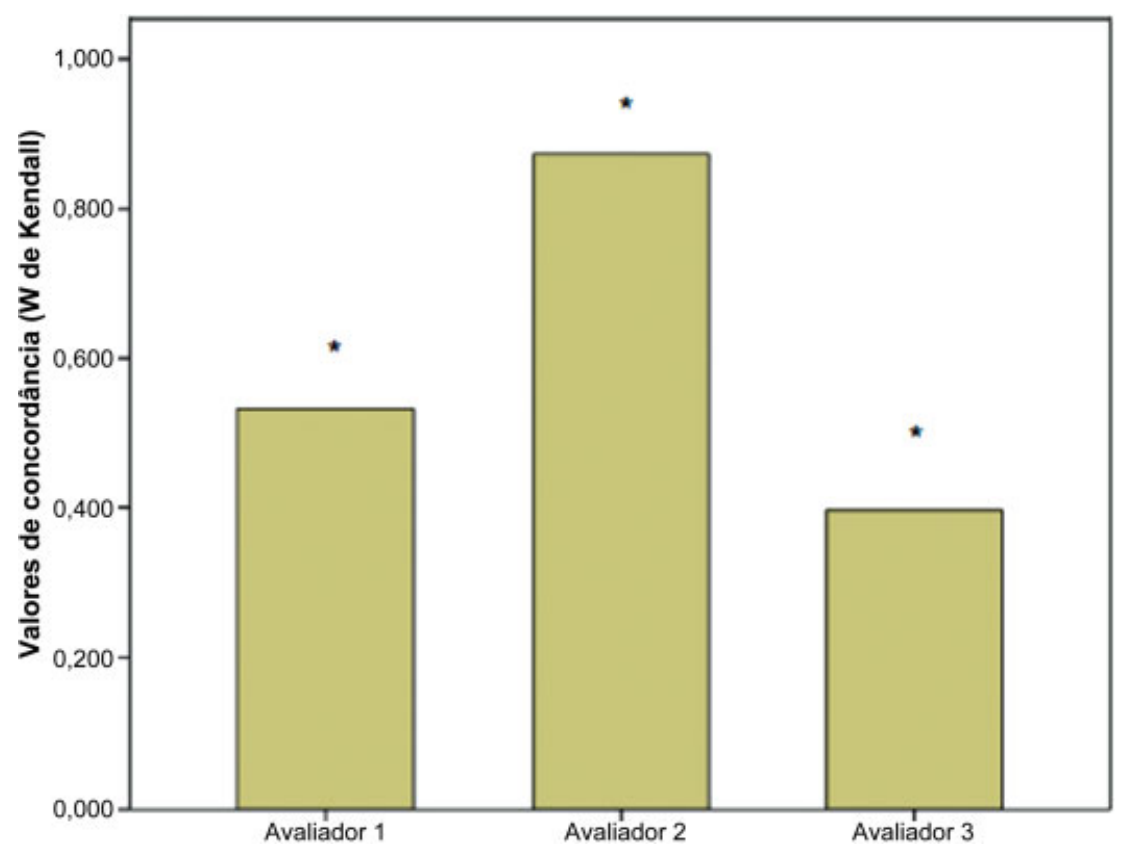

Fig. 3 Variação intraobservador $\left({ }^{*} \mathrm{p}<0,05\right)$.

3. Inclinação da patela: situação decorrente da insuficiência dos restritores mediais, a displasia da face patelar também desempenha um papel importante na sua producão;6,20

4. Patela alta: situação em que a patela, com o progredir da flexão, encaixa-se na face patelar femoral de forma instável, devido ao braço de alavanca alterado.6,20

O tratamento dos pacientes pode ser conservador ou cirúrgico, seguindo um fluxograma no qual vários fatores são levados em consideração ( - Fig. 4). ${ }^{18}$

Entre os principais fatores temos: número de luxações; falha ou não do tratamento conservador; distância TTLITAGT aumentada; inclinação patelar aumentada; patela alta; e a displasia troclear (quantificada pela classificação de Dejour e objeto de nosso estudo). ${ }^{18,20}$

Ou seja, para muitos autores a classificação em questão serve de guia para a condução clínica dos pacientes. Assim, é fundamental que a classificação em questão tenha uma boa reprodutibilidade.

Lippacher et $\mathrm{al}^{21}$ compararam a reprodutibilidade da classificação em 50 radiografias e 50 imagens de ressonância e chegaram à conclusão de que a classificação de Dejour é válida para a displasia, é particularmente útil na diferenciação entre as de baixo (A) e as de alto grau (B-D).

Rémy et $\mathrm{al}^{22}$ avaliaram apenas a reprodutibilidade da classificação com somente as radiografias em perfil e chega- ram à conclusão de que com apenas a radiografia a variação intra e interobservador apresenta baixa concordância.

Em estudo feito por Nelitz et al $^{23}$ é chamada a atenção para o valor limitado da classificação, é útil apenas para dividir em alto e baixo grau de displasia.

No presente estudo, fizemos uma análise em quatro cenários para classificar: com apenas a radiografia em perfil; com a radiografia e a tomografia; com a radiografia e a classificação no momento; e com a radiografia e a tomografia e a classificação no momento.

Como vimos nas figuras e - Tabela 2, mesmo consultando a classificação no momento, a variação interobservador com apenas a radiografia em perfil apresenta $W$ de Kendall não muito elevado. Todavia, as análises que usaram a radiografia e tomografia apresentaram coeficiente $\mathrm{W}$ de Kendall maior do que 0,8 , o que expressa excelente concordância.

Na avaliação intraobservador, de acordo com a - Tabela 1, um avaliador obteve excelente concordância, um regular concordância e o terceiro ruim concordância ( - Tabela 3 e - Fig. 3).

\section{Conclusão}

A classificação de Dejour gerou uma baixa reprodutibilidade intraobservador e interobservador quando usada somente a radiografia em perfil. 


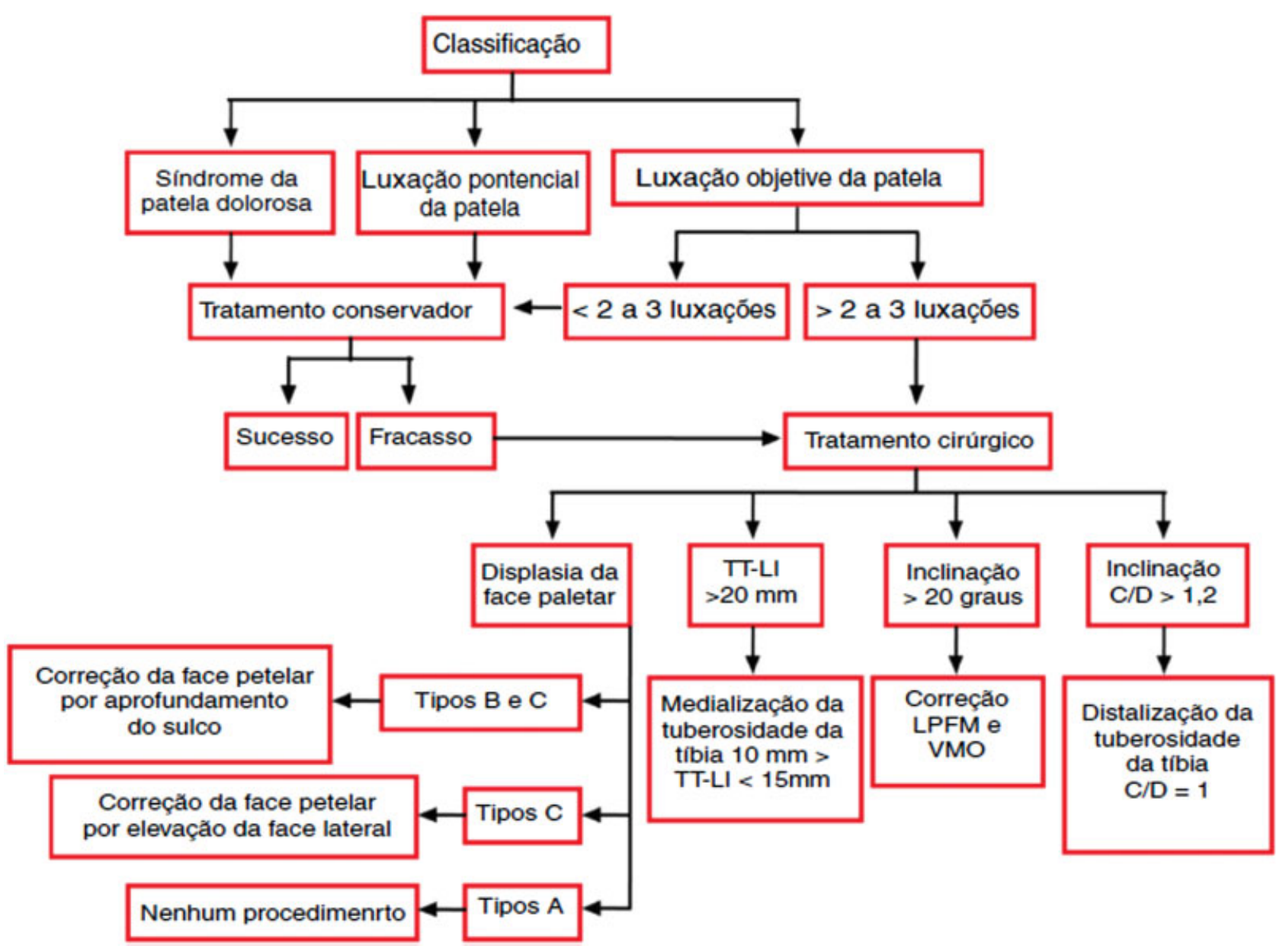

Fig. 4 Fluxograma para o tratamento da instabilidade femoropatelar. ${ }^{18}$

Ficou demonstrado que o uso de apenas a radiografia para classificar pode gerar falta de uniformidade até mesmo entre observadores experientes.

Contudo, quando usadas radiografia e tomografia associadas para classificar, a reprodutibilidade melhora.

Conflitos de interesse

Os autores declaram não haver conflitos de interesse.

\section{Referências}

1 Ribeiro MM, Nogueira F, Sardinha J, Jones JH. Critérios imagiológicos da instabilidade femoro-patelar por ressonância magnética. Rev Port Ortop Traumatol 2012;20(04):425-435

2 Ferrari D, Kuriki HU, Silva CR, Alves N, Mícolis de Azevedo F. Diagnostic accuracy of the electromyography parameters associated with anterior knee pain in the diagnosis of patellofemoral pain syndrome. Arch Phys Med Rehabil 2014;95(08):1521-1526

3 Rebouças Moreira TA, Demange MK, Gobbi RG, Mustacchi Z, Pécora JR, Passarelli Tírico LE, et al. Trochlear dysplasia and patellar instability in patients with Down syndrome. Rev Bras Ortop 2015;50(02):159-163

4 Fithian DC, Paxton EW, Stone ML, Silva P, Davis DK, Elias DA, et al. Epidemiology and natural history of acute patellar dislocation. Am J Sports Med 2004;32(05):1114-1121
5 Bollier M, Fulkerson JP. The role of trochlear dysplasia in patellofemoral instability. J Am Acad Orthop Surg 2011;19(01):8-16

6 Dejour H, Walch G, Nove-Josserand L, Guier C. Factors of patellar instability: an anatomic radiographic study. Knee Surg Sports Traumatol Arthrosc 1994;2(01):19-26

7 Steiner TM, Torga-Spak R, Teitge RA. Medial patellofemoral ligament reconstruction in patients with lateral patellar instability and trochlear dysplasia. Am J Sports Med 2006;34(08):1254-1261

8 White BJ, Sherman OH. Patellofemoral instability. Bull NYU Hosp Jt Dis 2009;67(01):22-29

9 Fulkerson JP. Diagnosis and treatment of patients with patellofemoral pain. Am J Sports Med 2002;30(03):447-456

10 Schöttle PB, Schmeling A, Rosenstiel N, Weiler A. Radiographic landmarks for femoral tunnel placement in medial patellofemoral ligament reconstruction. Am J Sports Med 2007;35(05):801-804

11 Gigante A, Pasquinelli FM, Paladini P, Ulisse S, Greco F. The effects of patellar taping on patellofemoral incongruence. A computed tomography study. Am J Sports Med 2001;29(01):88-92

12 Carrillon Y, Abidi H, Dejour D, Fantino O, Moyen B, Tran-Minh VA. Patellar instability: assessment on MR images by measuring the lateral trochlear inclination-initial experience. Radiology 2000; 216(02):582-585

13 Dejour H, Walch G. La pathologie femoro-patellaire. In: 6 émes Journees Lyonnaises de Chirurgie du Genou. Lyon, decembre. 1987

14 Dejour D, Reynaud P, Lecoultre B. Douleurs et instabilité rotulienne, Essai de classification. Med Hyg (Geneve) 1998;56(2217):1466-1471 
15 Burmann RC, Locks R, Pozzi JF, Konkewicz ER, Souza MP. Avaliac,ão dos fatores predisponentes nas instabilidades femoropatelares. Acta Ortop Bras 2011;19(01):37-40

16 Albuquerque RP, Giordano V, Sturm L, Azevedo Júnior V, Leão A, Amaral NP. Análise da reprodutibilidade de três classificações para a osteoartrose do joelho. Rev Bras Ortop 2008;43(08): 329-335

17 Gonc, alves FB, Rocha FA, Albuquerque RP, Mozella AP, Crespo B, Cobra H. Avaliac,ão da reprodutibilidade das diferentes descric, ões da classificac,ão de Kellgren e Lawrence para osteoartrite do joelho. Rev Bras Ortop 2016;51(06):687-689

18 Scott WN. Insall \& Scott surgery of the knee. Philadelphia: Churchill Livingstone; 2011

19 Azar FM, Canale ST, Beaty JH. Campbell's operative orthopaedics. Philadelphia: Elsevier Health Sciences; 2013
20 Scott WN. Insall \& Scott Surgery of the Knee. Philadelphia: Elsevier Health Sciences; 2017

21 Lippacher S, Dejour D, Elsharkawi M, Dornacher D, Ring C, Dreyhaupt J, et al. Observer agreement on the Dejour trochlear dysplasia classification: a comparison of true lateral radiographs and axial magnetic resonance images. Am J Sports Med 2012;40 (04):837-843

22 Rémy F, Chantelot C, Fontaine C, Demondion X, Migaud H, Gougeon F. Inter- and intraobserver reproducibility in radiographic diagnosis and classification of femoral trochlear dysplasia. Surg Radiol Anat 1998;20(04):285-289

23 Nelitz M, Lippacher S, Reichel H, Dornacher D. Evaluation of trochlear dysplasia using MRI: correlation between the classification system of Dejour and objective parameters of trochlear dysplasia. Knee Surg Sports Traumatol Arthrosc 2014;22(01):120-127 